\title{
Automated Negotiation for Opportunistic Energy Trading Between Neighbouring Wireless Sensor Networks
}

\author{
Andre P. Ortega ${ }^{1,2}$, Geoff V. Merrett ${ }^{1}$, Sarvapali D. Ramchurn ${ }^{1}$ \\ ${ }^{1}$ Electronics and Computer Science, University of Southampton, UK \\ ${ }^{2}$ Escuela Superior Politécnica del Litoral, Ecuador \\ apoa1g15@soton.ac.uk,gvm@ecs.soton.ac.uk,sdr1@soton.ac.uk
}

\begin{abstract}
As the Internet of Things grows, the number of wireless sensor networks deployed in close proximity will continue to increase. By nature, these networks are limited by the battery supply that determines their lifetime and system utility. To counter such a shortcoming, energy harvesting technologies have become increasingly investigated to provide a perpetual energy source; however, new problems arise as a result of their wide spatio-temporal variation. In this paper, we propose opportunistic energy trading, which enables otherwise independent networks to be sustained by sharing resources. Our goal is to provide a novel cooperation model based on negotiation to solve coordination conflicts between energy harvesting wireless sensor networks. Results show that networks are able to satisfy their loads when they agree to cooperate.

Index Terms-Ambient energy harvesting; autonomous sensors; Multiagent systems; agent-based sensor networks; opportunistic cooperation; energy negotiation.
\end{abstract}

\section{INTRODUCTION}

Internet of Things (IoT) deployments in industries, cities, healthcare and home automation are spread all over the world. A core technology required for IoT are wireless sensor networks (WSNs), which gather information from the environment, analyse it, make decisions and act accordingly. In many of these applications, sensor nodes are battery-powered and limited in energy supply. Thus, one proposed solution is to extend performance optimisation to the inter-network approach by enabling cooperation among networks that co-exist in a physical location [1]-[4].

Energy harvesting technologies have also gained widespread attention to enhance node lifetime. Moreover, ways to capture green energy from regenerative sources for self-sustainable operation is a key driver in today's low-power devices for smart applications. However, energy harvesting wireless sensor networks (EHWSNs) are conditioned to spatio-temporal variations of energy availability. The main objective of EHWSNs, because of their unlimited power supply, is the optimisation of their energy use to operate continuously. This mode of operation is called energy-neutral operation: a harvesting node achieves it if the energy supply during a harvesting period is sufficient to replace the amount consumed during the same time [5].

Adaptive algorithms have been developed to address the spatio-temporal variation of ambient energy sources and scale a node's performance appropriately, in order to deliver energyneutrality. These algorithms typically adjust parameters such as the duty-cycle or sampling rate [6], [7]. Other energyneutral algorithms exploit the spatial variation and distribute load according to energy reserves [8]. However, these algorithms are limited by the bounds of one network domain; i.e. if one node is expecting insufficient energy and the rest has a scarce energy input, no solution exists.

The cooperation problem among distinct WSNs has been studied in a game theoretic setting [1], [2]. These works model the behaviour of a network as a game to analyse the existence of strategies, looking for equilibrium among rational players that negotiate with each other to maximise their own benefit. They focus on the conditions under which cooperation is the best strategy in multi-domain WSNs, and make an exhaustive search on the available space to find a solution for each network's authority (i.e. those that form a Nash equilibrium with the highest possible lifetimes). For a WSN, this would necessitate nodes making a significant effort to calculate and store not only all their possible actions at each decision point, but also the ones corresponding to the other nodes. This is not feasible for devices with limited memory and power. One approach to deal with this complexity is to simplify

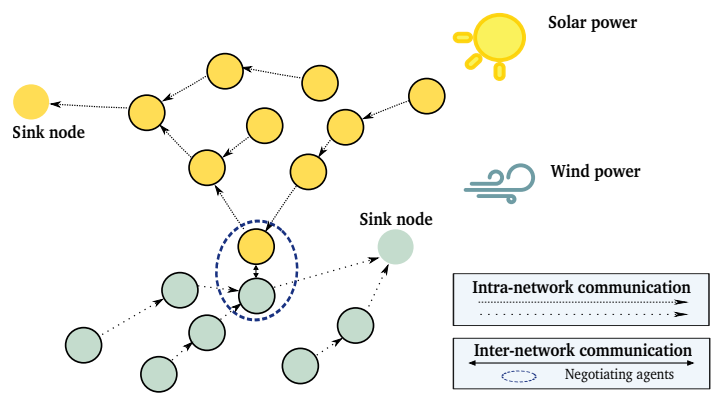

Fig. 1: Direct interconnection between co-located EHWSNs.

the settings in which nodes interact with each other and use heuristic methods. Before cooperation can be established, networks should be able to interact and find a mutuallyacceptable agreement in favour of maximising their utilities. In the domain of EHWSNs, they must find an energy flow that deals with the spatio-temporal profile of their energy sources and satisfies as much as possible their energy consumption 
profile from collaborative effort. A multi-agent approach is a natural fit to this setting as individual sensor nodes need to autonomously negotiate and form an agreement as to how to share their resources [9], [10].

Against this background, we motivate the use of a solution based on automated negotiation and propose a novel approach to model cooperation between nodes with a direct interconnection architecture, i.e. without an intermediary (Figure 1). The contributions of the work reported in this paper are:

- An alternating offers protocol for the nodes to exchange offers to trade energy-hungry services.

- An optimisation algorithm based on Linear Programming (LP) to optimise the allocation of energy to maximise individual actors' preferences.

- An analysis of time-dependent negotiation strategies in EHWSNs for energy re-allocation of distinct energy harvesting sources.

- Results showing how negotiation can be delimited by a short-term deadline and end in social-welfare maximising deals.

\section{System Model AND PRoblem Formulation}

We assume an initial simplified setting where two distinct EHWSNs with a different type of energy harvesting source (e.g. solar and wind) share the same location and direct interconnection is possible between each pair of nodes with overlapping radio range [11]. Our main motivation in investigating negotiation applied in this domain is to observe the effects of cross-boundary energy transfer for sensor's power management and this setting is suitable for that purpose.

While it is convenient to envisage opportunistic energy trading as physically transferring energy across a network boundary, energy is actually logically transferred by accepting energy-consuming tasks as data processing or packet forwarding [1]-[3]. For example, each network involved may control the agreed energy flow by asking for/providing routing favours.

\section{A. Model Assumptions}

Each network $N_{k}, k \in\{1,2\}$ is formed by a set of unique sensor nodes and a sink. Each node is controlled by an agent, which is denoted as $\alpha_{k, i}, i \in \mathbb{N}$. The agent has complete knowledge of all the relevant node's information, such as its energy profile variables, battery capacity and residual energy.

We assume that the period at which energy is harvested by a node is $T$ (e.g. 24 hours for solar energy) and it is divided into discrete time slots $T=(1, \ldots, n)$ of equal duration $L$.

\section{B. Energy Consumption Model}

Each node controlled by $\alpha_{k, i}$ consists of an energy harvester unit, a rechargeable battery and several loads: a radio, CPU and sensors. We use the energy model introduced in [12] and define $\boldsymbol{E}_{\boldsymbol{k}, \boldsymbol{i}}^{\boldsymbol{c}}=\left(E_{k, i}^{c}(1), \ldots, E_{k, i}^{c}(n)\right): \boldsymbol{E}_{\boldsymbol{k}, \boldsymbol{i}}^{\boldsymbol{c}} \in \mathbb{R}^{+}$as the energy consumed by $\alpha_{k, i}$ over $n$ time slots. At any given slot $t$, we can calculate the total energy $E_{k, i}^{c}$ that an agent $\alpha_{k, i}$ consumes as:

$$
E_{k, i}^{c}(t)=V \cdot\left[D \cdot I^{\text {active }}+(1-D) \cdot I^{\text {sleep }}\right] \cdot L
$$

Then, the energy is dependent on the duty cycle $D$, supplied voltage $V$, active mode current $I^{\text {active }}$ and sleep mode current $I^{\text {sleep }} . D$ is chosen by the application, while $I^{\text {active }}, I^{\text {sleep }}$ and $V$ can be known in advance using datasheet information.

\section{Energy Management Model}

Our model is built on the models proposed by [5] and [13]. We assume that all nodes can harvest energy and store it in their battery for future use. Without loss of generality, we assume that the replenishment of energy occurs at the beginning of each time slot $t$.

The expected energy input during each slot $t$ can be forecast from historical information with a high level of accuracy. Energy can then be allocated to each slot $t$. We use $E_{k, i}^{c}(t)$ and $E_{k, i}^{h r v}(t)$ to denote the energy profile variables for each time slot. The amount of energy that can be generated by the harvesting unit in $n$ time slots is defined as $\boldsymbol{E}_{\boldsymbol{k}, \boldsymbol{i}}^{\boldsymbol{h r} \boldsymbol{v}}=$ $\left(E_{k, i}^{h r v}(1), \ldots, E_{k, i}^{h r v}(n)\right): \boldsymbol{E}_{\boldsymbol{k}, \boldsymbol{i}}^{\boldsymbol{h r} \boldsymbol{v}} \in \mathbb{R}^{+}$. For example, if the harvesting period starts at 00:00 and $L$ is 1 hour, then $E_{k, i}^{h r v}(1)$ is the expectation for the energy harvested during slot 1 (from 00:00 to 01:00), $E_{k, i}^{h r v}(2)$ is the expectation of energy during slot 2 (01:00 to 02:00), etc.

$B_{k, i}(t)$ is used to represent the residual battery energy at the beginning of slot $t$ in agent $\alpha_{k, i}$. Then $\boldsymbol{B}_{\boldsymbol{k}, \boldsymbol{i}}=$ $\left(B_{k, i}(1), \ldots, B_{k, i}(n)\right): \boldsymbol{B}_{\boldsymbol{k}, \boldsymbol{i}} \in \mathbb{R}^{+}$denotes the battery level in $n$ time slots. The battery is characterised by a limited capacity $B_{k, i}^{\max }$ and charging efficiency $\eta$. The battery enables an agent to save and use energy throughout a day, which helps the agent to compute an energy allocation, $\boldsymbol{E}_{\boldsymbol{k}, \boldsymbol{i}}^{\text {alloc }}=$ $\left(E_{k, i}^{\text {alloc }}(1), \ldots, E_{k, i}^{\text {alloc }}(n)\right): \boldsymbol{E}_{\boldsymbol{k}, \boldsymbol{i}}^{\text {alloc }} \in \mathbb{R}^{+}$, to assign the harvested energy $E_{k, i}^{h r v}$ to the energy consumed $E_{k, i}^{c}$ by the load of the node.

When $E_{k, i}^{h r v}(t)$ is lower than $E_{k, i}^{c}(t)$, some of the energy used by the sensor node is discharged from the battery. We use $\boldsymbol{d}=(d(1), \ldots, d(n)): \boldsymbol{d} \in \mathbb{R}^{+}$to represent this amount. When $E_{k, i}^{h r v}(t)$ is higher than $E_{k, i}^{c}(t)$, all the energy used in the node is provided by the energy source and the battery is charged with the excess, as required. We use $\boldsymbol{c}=(c(1), \ldots, c(n))$ : $c \in \mathbb{R}^{+}$to denote this amount in $n$ time slots. Any excess energy received at times when the battery is full is discarded by the node. The energy that the agent is unable to use or store is waste, denoted by $\boldsymbol{w}_{\boldsymbol{k}, \boldsymbol{i}}=\left(w_{k, i}(1), \ldots, w_{k, i}(n)\right): \boldsymbol{w}_{\boldsymbol{k}, \boldsymbol{i}} \in \mathbb{R}^{+}$. Then we can calculate the energy used from the battery in any slot $t$ as:

$$
B_{k, i}(t)-B_{k, i}(t+1)=d(t)-\eta \cdot c(t)
$$

In our domain, an opportunistic energy trade is triggered when a node's energy level has dropped below a threshold. Then, the initial battery status $B_{k, i}(1)$ is equal to $\eta \cdot b$ where $b$ is the energy level at $t=1$. At each time $t, \alpha_{k, i}$ also considers the amount of energy to receive/give from the negotiation, which is defined by $\boldsymbol{o}=(o(1), \ldots, o(n)): \boldsymbol{o} \in \mathbb{R}^{+}$. o represents the offer of energy at each time slot, i.e. The issues of this negotiation domain. We call these offers energy flow offers. A valid energy flow offer must include the energy values for the predetermined time of cooperation, e.g. If networks expect to cooperate for 24 hours, then the energy flow must 
include 24 values. The direction of the energy flow is denoted by a positive or negative sign. If positive, the amount is an offer of energy from the agent to its opponent, otherwise, it represents the energy to be received from the opponent. For example, if two agents are willing to cooperate with each other for a period of 2 hours and $L$ is set to 30 minutes, then an offer of energy from agent $\alpha_{k, i}$ to the other party can be $o=[-1.88,-0.7,18,-4]$; where $-1.88 \mathrm{mWh},-0.7 \mathrm{mWh}$ and $-4 \mathrm{mWh}$ represent the energy savings of $\alpha_{k, i}$ from the opponent's cooperation (e.g. by packet routing) at time slots 1,2 and 4 respectively, while $\alpha_{k, i}$ compromises to provide 18 $\mathrm{mWh}$ through collaborative effort to its opponent at time slot 3.

\section{Utility Function}

The objective function of this model is described as the total energy consumption that is satisfied (i.e. energy allocation $\left.E_{k, i}^{\text {alloc }}\right)$ at period $T$. Then the utility of an agent represented by $u$ is defined as follows:

$$
\text { Objective } \max u=\sum_{t=1}^{n} E_{k, i}^{\text {alloc }}(t)
$$

Subjected to the following constraints:

$$
\begin{gathered}
E_{k, i}^{\text {alloc }}(t)=E_{k, i}^{h r v}(t)-c(t)+d(t)+o(t)-w(t) \\
E_{k, i}^{\text {alloc }}(t) \leq E_{k, i}^{c}(t) \\
B_{k, i}(t)-B_{k, i}(t+1)=d(t)-\eta \cdot c(t) \\
B_{k, i}(1)=\eta \cdot b \\
0 \leq c(t) \leq B_{k, i}^{\max } \\
E_{k, i}^{c}(t)-E_{k, i}^{h r v}(t) \leq d(t) \leq B_{k, i}^{\max } \\
0 \leq B_{k, i}(t) \leq B_{k, i}^{\max } \\
0 \leq w(t) \leq E_{k, i}^{\text {hrv }}(t)
\end{gathered}
$$

Equations (c1) and (c2) represent the energy balancing condition. The allocated energy to a node defined by the harvested energy, battery flow, the energy offer and waste is equal or smaller than the node's load at time slot $t$. Equations (c3)(c7) define the battery status and flows constraints regarding its capacity. Equation (c8) is used to guarantee that the energy waste is an excess of the energy harvested.

The solution to the optimisation problem yields the amount of energy that must be allocated to a sensor node in every $t$ and the evolution of residual energy in its battery over period $T$. Following the model described, an agent can compute the optimal energy flow that benefits both agents, but this requires complete information and high computation capabilities since the set of all possible agreements is exponential in the number of time slots. Cooperative approaches must ideally result in Pareto-efficient outcomes, which means that one agent cannot be better off without making the other agent worse off. In section IV, we present a cooperative solution that satisfies this property of efficiency known as the Nash Bargaining Solution (NBS) [14] to find an agreed energy flow between agents, but first, we describe the heuristic model used for the bargaining process of this domain.

\section{HEURISTIC APPROACH FOR OPPORTUNISTIC ENERGY} TRADING

There are four fundamental parts in a negotiation model described by a heuristic approach: 1) the negotiation protocol or rules of interaction for the agents, 2) the definition of issues or objects in contention (see II-C), 3) the utility function or agents' preference model (see II-D), and 4) the tactics or offers' generator functions that are applied during the bargaining process, which along with the utility function comprise the decision making apparatus the participants employ to act according to the negotiation protocol and reach their desired goals [15], [16]. The protocol and tactic employed are defined below.

\section{A. Multi-issue Bilateral Negotiation Protocol}

We adopt Rubinstein's alternating-offers protocol [17] for the negotiation of energy among neighbouring EHWSNs. In a bilateral negotiation, both agents desire to cooperate but have conflicting interests regarding their preferences (in this domain due to distinct batteries, power consumption and energy harvesting profiles).

According to the protocol, all the agents involved have one turn per round to respond to the current state of the negotiation. One of the negotiating agents starts with an offer to its opponent. Whenever an offer is made, the opponent can accept or reject the offer. If the offer is accepted, then the bargaining ends and an agreement is reached. If the offer is rejected, the agent in turn proposes an agreement, which again the opponent may accept or reject in the next round. We continue the negotiation until a final negotiation round. When one negotiating agent reaches a final round without a favorable response or an agreement is found, the negotiation ends. In the first case, the negotiation fails and terminates with no deal possible.

In our domain, we must consider the number of messages exchanged between nodes and limit the negotiation to a shortterm deadline. Thus, a predefined maximum negotiation round is set. Specifically, in our scenario, automated negotiation can complete in seconds, which makes time inappropriate to model the deadline. In each negotiation round, an offer contains multiple issues that are negotiated simultaneously. We assume that the knowledge of the negotiation domain (issues, deadline, initial negotiating agent) is known by both agents beforehand, and is not changed during the whole negotiation process. As defined in II-C, o represents the offer of energy. Thus, $o_{1,1 \rightarrow 2,1}^{r}$ is a vector of values proposed by agent $\alpha_{1,1}$ to agent $\alpha_{2,1}$ at round $r$, where $o_{1,1 \rightarrow 2,1}^{r}(t)$ is the value of energy proposed from $\alpha_{1,1}$ to $\alpha_{2,1}$ for time $t$. Each issue $o(t)$ has an acceptable range of values represented as the interval $\left[\min _{k, i} o(t), \max _{k, i} o(t)\right]$.

\section{B. Negotiation tactic}

In the negotiation context, heuristics are useful for the generation of initial offers, evaluation of proposals and decision of counter offers, based on computational approximations that produce good close to Pareto-efficient outcomes. 
The main advantage of using heuristics in this domain is to model encounters between networks that are discovered opportunistically and have no information about the resources and preferences of each other.

Faratin et al. [15] studied strategic negotiation between autonomous computational agents and develop a formal model of reasoning to address the coordination problem. They defined a number of heuristic functions, which receive the name of tactics and use a single criterion (time, resources, behaviour, etc.) to generate new values for each issue in the negotiation set. The following family of tactics for counter-offer generation were applied in this domain.

1) Time-dependent tactics (TDT): The time elapsed in the negotiation is what conducts the values of the negotiation issues. It is the same for rounds, the more rounds has passed the more pressure is induced and faster concessions are possible. Then the value of $o(t)$ proposed by agent $\alpha_{1,1}$ to agent $\alpha_{2,1}$ at round $r$ is giving by the following equation:

If $\alpha_{1,1}$ 's utility decreases with issue $o(t)$ :

$$
o_{1,1 \rightarrow 2,1}^{r}(t)=\min _{1,1} o(t)+\gamma_{o(t)}^{r}\left(\max _{1,1} o(t)-\min _{1,1} o(t)\right)
$$

If $a$ 's utility increases with issue $o(t)$ :

$o_{1,1 \rightarrow 2,1}^{r}(t)=\min _{1,1} o(t)+\left(1-\gamma_{o(t)}^{r}\right)\left(\max _{1,1} o(t)-\min _{1,1} o(t)\right)$

We define $\gamma_{o(t)}^{r}$ as a polynomial function parameterised by $\beta \in \mathbb{R}_{n}^{+}$as follows:

$\gamma_{o(t)}^{r}=k_{1,1} o(t)+\left(1-k_{1,1} o(t)\right)\left(\min \left(r, \operatorname{rmax}_{1,1}\right) / \operatorname{rmax}_{1,1}\right)^{1 / \beta}$.

The constant $k$ at $t=1$ represents the initial bargaining value of $o(t)$ while rmax is the deadline. $\beta_{>0}$ defines the convexity degree of the curve. When $\beta>1$, the agent is benevolent and characterised by a conceder behaviour (such tactic is called Conceder) and the offer rapidly changes to the reservation value. At $0<\beta<1$, the agent is tough and maintains its initial offer until it almost approaches the deadline (such tactic is known as Boulware). We limit our examinations to these negotiation tactics, while behavioural heuristics would be more appropriate in a dynamic environment as EHWSNs. But, these are less successful in short-term deadlines [15].

\section{NUMERICAL ANALYSIS}

We assume agents observe that their residual energy level has dropped below the threshold set, they are appropriately synchronised and plan to cooperate for the next 24 hours, which start at 00:00 and end at 23:00 local time with $L=1$ hr, i.e. agents negotiate an energy flow of 24 values. Then $T$ corresponds to the same period and time slots $T=(1, \ldots, 24)$. Numerical results are shown to demonstrate the performance of the agents with trading over the individualistic approach. All the results are obtained using MATLAB.

\section{A. Simulation Setup}

In this section, we study the problem of cooperation in a simplified scenario with a pair of nodes (each from a different network); negotiating agents $\alpha_{1,1}$ and $\alpha_{2,1}$ of $N_{1}$ and $N_{2}$, respectively.

Agent $\alpha_{1,1}$ is simulated as controlling a Memsic eKo mote, containing a $3.3 \mathrm{~cm} \times 6.35 \mathrm{~cm}$ photovoltaic cell (assumed to be $10 \%$ efficient) to recharge a $600 \mathrm{mAh}$ battery. We consider $\eta=0.7$, which is typical of NiMH batteries. Agent $\alpha_{2,1}$ is simulated as controlling a Memsic MICAz node, with a micro-wind turbine to recharge a $600 \mathrm{mAh}$ battery. The energy model in II-B is used to evaluate the energy consumption of both agents, using parameters obtained from empirical measurements and datasheets [18]. We consider a realistic scenario where an eKo node operates at $1 \%$ duty cycle, and the average power consumption is $0.615 \mathrm{~mW}$. For the MICAz mote, an average load of $2.86 \mathrm{~mW}$ at $5 \%$ duty cycle of operation is expected. Thus, agent $\alpha_{1,1}$ and $\alpha_{2,1}$ demand 0.615 $\mathrm{mWh}$ and $2.86 \mathrm{mWh}$ of energy in each time slot, respectively.
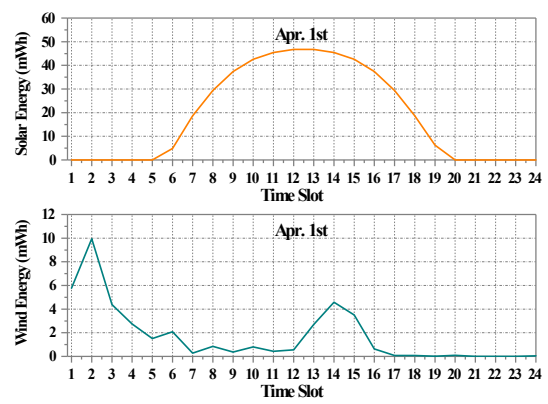

Fig. 2: Solar (agent $\alpha_{1,1}$ ) and wind energy (agent $\alpha_{2,1}$ ) harvested throughout a day.

The meteorological information used to compute the energy generation corresponds to the area of Southampton, UK $\left(50.8997^{\circ} \mathrm{N},-1.3955^{\circ} \mathrm{W}\right.$, Elevation $\left.32 \mathrm{~m}\right)$ [19], [20].

The values of solar irradiance from April 2017 are used to estimate the hourly power output of a photovoltaic system for a day, which is proportional to the solar radiation, the panel dimension, and its efficiency. The estimated hourly energy output is shown in Figure 2. The energy exhibits a temporal variation that favours time slots 6-19 which correspond to times 05:00-18:00. The total energy generated is $452 \mathrm{mWh}$.

We adopt daily data from April 2017 to estimate the hourly average wind speed for a day. The power from the wind source can be calculated from its speed as in [21] considering a swept area of $5 \mathrm{~cm} \times 5 \mathrm{~cm}$ for the wind turbine. From April data, we chose April 1st. The diversity between generation times in solar and wind creates an opportunity for energy trading. Then we scale this data to get the hourly power output of a highly efficient micro-turbine (Figure 2). The total energy generated in a day is $41.4 \mathrm{mWh}$.

\section{B. Results}

With the nodes' information and energy profiles described above, agents can compute their utilities (without trading) using the LP model described in Section II-D when the offer $o$ is null. We compare the utility achieved by the agents without trade, by NBS and by the bilateral negotiation protocol using TDT. Figures 3 and 4 for agents $\alpha_{1,1}$ and $\alpha_{2,1}$ respectively, 

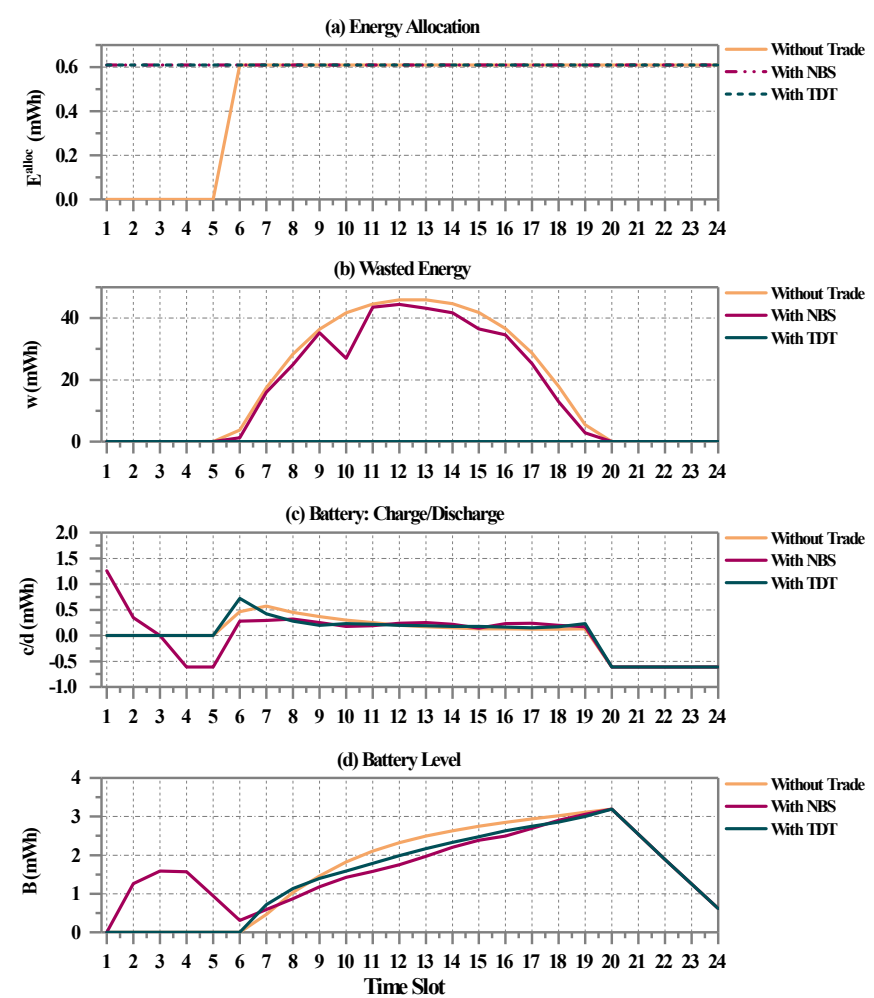

Fig. 3: Agent $\alpha_{1,1}$ : Results of utility maximisation without trade, with NBS and with TDT.

show how agents can increase their utilities via cooperation and reduce the waste of energy excess. As presented in Figures 3.(a) and 4.(a), the energy allocation without trade is insufficient at time slots 1-5 for agent $\alpha_{1,1}$ and 10-13, 1724 for $\alpha_{2,1}$, while it is equal to the load when there is energy trading. Thus, $u$ for $\alpha_{1,1}$ increases from 0.79 to 1 and 0.59 to 1 for agent $\alpha_{2,1}$ when they reach an agreement to cooperate. The achievement of energy-neutrality in both scenarios depends in this case on the amount of unused energy from both agents and their matching requirements.

The results shown of TDT are obtained for a negotiation deadline set to 10 rounds. At the beginning of the negotiation, the agents make the offers that give the highest utility to themselves. No matter how low or high we vary the concession shape $\beta$ ( 0.5 or 1.8 ) for any agent, the negotiation process with TDT ends with these results. If agent $\alpha_{1,1}$ starts, the process ends in the first round, otherwise it ends in the second round after $\alpha_{2,1}$ agrees with the counter offer of agent $\alpha_{1,1}$. Agent $\alpha_{1,1}$ has a large excess of energy to offer that satisfies agent $\alpha_{2,1}$ requirements (Figure 3.(b) Without Trade) and $\alpha_{2,1}$ is also able to assist $\alpha_{2,1}$ in its lack of energy during periods $1-5$. In result, the utilisation of energy is maximised from $52.2 \mathrm{mWh}$ to $83.3 \mathrm{mWh}$ by negotiation while maintaining the application performance at the same rate at all times, i.e. the duty cycle is not affected. Then, the total energy saved via cooperation can be up to $7.08 \%$ for one day of the energy generated. The energy saved
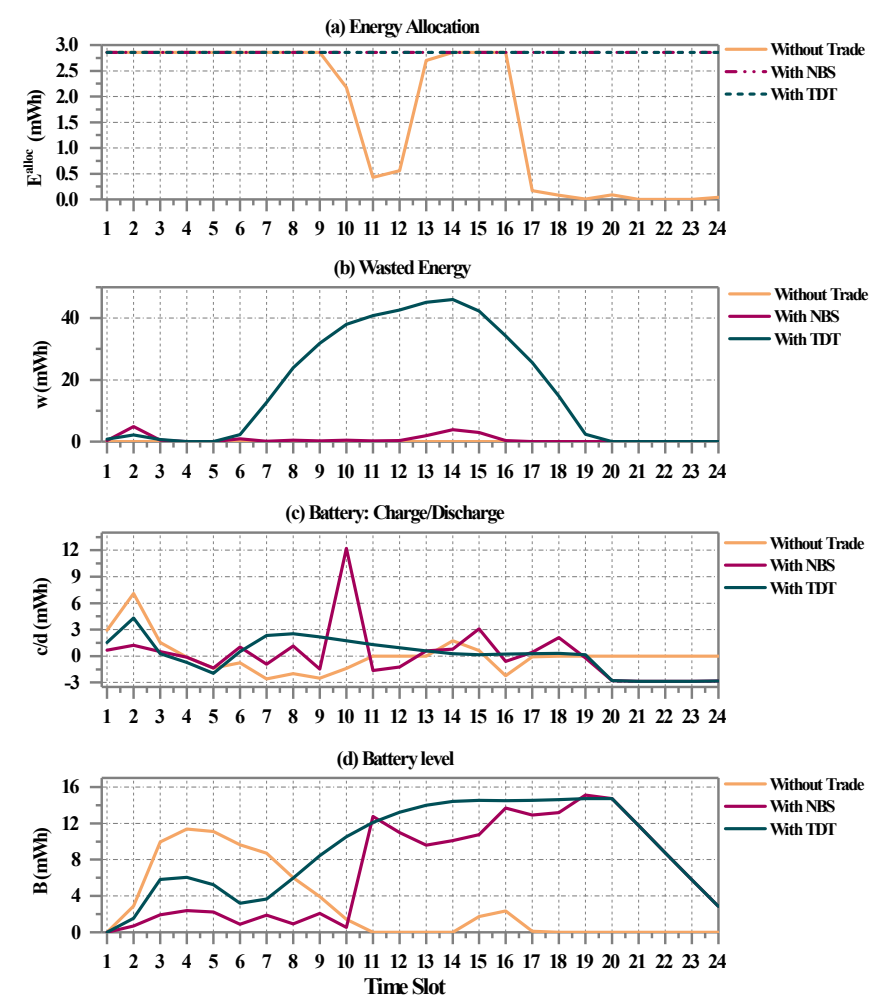

Fig. 4: Agent $\alpha_{2,1}$ : Results of utility maximisation without trade, with NBS and with TDT.

corresponds to the energy reallocated in cooperation which would otherwise go to waste without trade. The reduction of energy waste is illustrated in Figures 4.(b) Without Trade and Figure 4.(b) with TDT. Figures 3.(c), 3.(d), 4.(c) and 4.(d) show the state of the battery during the day for each agent, where the battery level matches the dynamics of the charging and discharging flows and none exceeds the maximum battery capacity. The difference in the battery dynamic between NBS and TDT depends on the negotiation's final outcome. For NBS, the solution corresponds to the offer $o_{1,1 \rightarrow 2,1}=$ $[-1.88,-0.96,-0.62,0,0,2.75,1.78,3.57,1.23,14.77,1.07$, $1.37,2.7,2.88,5.33,1.94,3.25,4.91,2.64,0,0,0,0,0]$

while TDT finishes in $o_{1,1 \rightarrow 2,1}=$ $[-0.61,-0.61,-0.61,-0.61,-0.61,3.54,17.61,28.49,36.59$ , 41.75, 44.59, 45.91, 45.92, 44.63, 41.8, 36.63, 28.63, 17.86, $5.39,0,0,0,0,0]$, which represent the 24 energy values (in $\mathrm{mWh}$ ) agreed for a day of cooperation.

To evaluate the proposed cooperation model and compare the different agent behaviours, we make a slight change and match the load of agent $\alpha_{1,1}$ to agent $\alpha_{2,1}$. The results are shown in Table I as the agent that starts the negotiation, who finishes it, behaviours, utilities and final round. The following cases are considered:

- Case 1: Both agents employ a Conceder tactic.

- Case 2: Both agents employ a Boulware tactic.

- Case 3: $\alpha_{1,1}$ is tough while $\alpha_{2,1}$ concedes.

- Case 4: $\alpha_{1,1}$ concedes while $\alpha_{2,1}$ is tough. 
TABLE I: Comparison between different negotiation cases

\begin{tabular}{|c|c|c|c|c|c|}
\hline First turn & Final turn & Case & $\mathrm{u} \alpha_{1,1}$ & $\mathrm{u} \alpha_{2,1}$ & Final round \\
\hline$\alpha_{1,1}$ & $\alpha_{2,1}$ & 1 & 0.93 & 1 & 2 \\
$\alpha_{2,1}$ & $\alpha_{2,1}$ & 1 & 1 & 0.94 & 2 \\
$\alpha_{1,1}$ & $\alpha_{2,1}$ & 2 & 0.97 & 0.96 & 7 \\
$\alpha_{2,1}$ & $\alpha_{2,1}$ & 2 & 0.97 & 0.96 & 8 \\
$\alpha_{1,1}$ & $\alpha_{2,1}$ & 3 & 1 & 0.93 & 2 \\
$\alpha_{2,1}$ & $\alpha_{2,1}$ & 3 & 1 & 0.94 & 2 \\
$\alpha_{1,1}$ & $\alpha_{1,1}$ & 4 & 0.91 & 1 & 3 \\
$\alpha_{2,1}$ & $\alpha_{1,1}$ & 4 & 0.91 & 1 & 3 \\
\hline
\end{tabular}

In those situations, energy-neutrality is only accomplished by one agent at a time and only when the opponent is benevolent. We can see that it is not possible to satisfy any energy consumption profile if both agents adopt a tough negotiation strategy. Similar to our simulation result before, the required number of rounds is low. The highest energy utilisation is given whenever agent $\alpha_{2,1}$ concedes faster at the beginning of the negotiation and while it has the first turn. In both cases, agent $\alpha_{1,1}$ reaches energy-neutrality. The second result of the table matches the utility levels reached by the optimal solution if a central and trustable authority is available to collect the information about the agents and calculate NBS. Since most of the parameters are the same for both agents (except the energy harvested), the available energy is a decisive factor in the establishment of cooperation. When sensors are energy-aware, spontaneous cooperation cannot take place and thus, a negotiation is required.

The presented results provide some insight on cooperation initiated by a negotiation, but more simulations have to be conducted to evaluate the model. For example, in our scenarios, additional costs for energy re-allocation, e.g. due to offers exchange, are not yet considered. Such issues, as well as further investigation in the effect of the network's dynamism on the negotiation model, is required.

\section{CONCLUSions AND Future WORK}

In this paper, we have proposed a model of EHWSNs and also applied a negotiation heuristic based on a TDT to optimally allocate the harvested energy by the nodes involved at each time slot. The main advantage of this cooperation initiated by a negotiation is that it allows to establish an opportunistic interaction between networks that cannot be conceived at design time about the resources of their neighbours, leading to a more integrated system of EHWSNs, while at the same time the use of the energy harvested is maximised.

The vision of WSNs cooperation brings many implications (from protocol diversity to security concerns), where several steps must be taken in order to ensure an effective interaction. One of the main challenges in extending power management to an area wider than the boundaries of one domain is the heterogeneity in terms of resources, which is the problem addressed in this work. An essential factor to establish cooperation is to know the costs and benefits that will incur to the parties. Here, a negotiation approach has been evaluated as a mechanism for networks to communicate and compromise to reach mutually beneficial results. In the future, we expect to extend the model to consider multiple nodes and the uncertainty generated by the energy availability and unexpected weather conditions.

\section{ACKNOWLEDGMENT}

This research is supported by Secretaría de Educación Superior, Ciencia, Tecnología e Innovación (SENESCYT) and Escuela Superior Politécnica del Litoral (ESPOL) under Ph.D. studies 2016, and the UK EPSRC under grant EP/P010164/1. Data supporting the results presented in this paper is available at https:/doi.org/10.5258/SOTON/D0621.

\section{REFERENCES}

[1] Levente Buttyán et al. Spontaneous cooperation in multi-domain sensor networks. In European Workshop on Security in Ad-hoc and Sensor Networks, pages 42-53, 2005.

[2] Márk Félegyházi et al. Cooperative packet forwarding in multidomain sensor networks. In Pervasive Computing and Communications Workshops, pages 345-349, 2005.

[3] Teng Jiang et al. Opportunistic energy trading between co-located energy-harvesting wireless sensor networks. In Proc. of the 1st International Workshop on Energy Neutral Sensing Systems, page 11, 2013.

[4] Eli De Poorter et al. A negotiation-based networking methodology to enable cooperation across heterogeneous co-located networks. Ad Hoc Networks, vol. 10(6):901-917, 2012.

[5] Aman Kansal et al. Power management in energy harvesting sensor networks. ACM Transactions on Embedded Computing Systems, vol. 6(4):32, 2007.

[6] Christian Renner et al. Adaptive energy-harvest profiling to enhance depletion-safe operation and efficient task scheduling. Sustainable Computing: Informatics and Systems, vol. 2(1):43-56, 2012.

[7] Jason Hsu et al. Adaptive duty cycling for energy harvesting systems. In Proc. of the 2006 International Symposium on Low power electronics and design, pages 180-185.

[8] Zhi Ang Eu et al. Opportunistic routing in wireless sensor networks powered by ambient energy harvesting. Computer Networks, vol. 54(17):2943-2966, 2010.

[9] Victor Lesser et al. Distributed sensor networks: A multiagent perspective, volume vol. 9. 2012.

[10] Meritxell Vinyals et al. A survey on sensor networks from a multiagent perspective. The Computer Journal, vol. 54(3):455-470, 2011.

[11] Teng Jiang et al. Opportunistic direct interconnection between co-located wireless sensor networks. In Int. Conf. on Computer Communications and Networks, pages 1-5, 2013.

[12] Xiaofan Jiang et al. Perpetual environmentally powered sensor networks. In Proc. of the 4th international symposium on Information processing in sensor networks, page 65, 2005.

[13] Alam Muddasser et al. A negotiation protocol for multiple interdependent issues negotiation over energy exchange. In Proc. of the AI for an Intelligent Planet, page 1, 2011.

[14] John F. Nash Jr. The bargaining problem. Econometrica: Journal of the Econometric Society, pages 155-162, 1950.

[15] Peyman Faratin et al. Negotiation decision functions for autonomous agents. Robotics and Autonomous Systems, vol. 24(3-4):159-182, 1998.

[16] Nicholas R. Jennings et al. Automated negotiation: prospects, methods and challenges. Group Decision and Negotiation, vol. 10(2):199-215, 2001.

[17] Ariel Rubinstein. Perfect equilibrium in a bargaining model. Econometrica: Journal of the Econometric Society, pages 97-109, 1982.

[18] MEMSIC Corporation, Inc. http://www.memsic.com/. Accessed on March 01, 2018.

[19] Southampton Weather. http://www.southamptonweather.co.uk/wxabout. php. Accessed on March 01, 2018.

[20] PVGIS. http://re.jrc.ec.europa.eu/pvgis/apps4/pvest.php. Accessed on March 01, 2018.

[21] Shad Roundy et al. Power sources for wireless sensor networks. In European workshop on wireless sensor networks, pages 1-17, 2004. 